\title{
Energy consumption forecast and charging demand alert based on operation condition clustering and control variable method
}

\author{
Xuejing Huang ${ }^{1}$, Jun $\mathrm{Jia}^{2, \mathrm{a}}$, Wei Xiao ${ }^{2}$, Ji Zhao ${ }^{2}$, Zhe Chen ${ }^{1}$, Zongyang Liu ${ }^{1}$, Jiaqi Li ${ }^{1}$ \\ ${ }^{1}$ China Southern Power Grid Dongguan Power Supply Bureau, 523003 Dongguan, Guangdong \\ 2 Tsinghua Sichuan Energy Internet Research Institute, 610042 Chengdu, Sichuan
}

\begin{abstract}
Travel anxiety of automobile owners has been aggravated because of the difficulty in accurately controlling the operation energy consumption and imperfection in charging infrastructure construction and other problems. Relying on the massive historical operation data of automobiles, it acquired the powerconsumption increasing coefficient of speed and temperature by means of clustering and control variable methods. Furthermore, the map Application Programming Interface (API) was invoked to obtain the path planning results thus realizing prediction on power consumption. The historical charging data of current automobile was used to build the mapping relations of the state of charge (SOC) and the state of energy (SOE). Combining with the prediction value of energy consumption it calculated the needed charge capacity and judge whether to issue the charging demand alert. Indicated by the application results, the proposed algorithm of energy-consumption forecast is more accurate than traditional average energy-consumption forecast algorithm. Accordingly, the charging demand alert function can effectively relive the travel anxiety of automobile owners.
\end{abstract}

\section{Introduction}

"The Development Scheme of New Energy Automobile Industry (2021-2035) [1]" clearly specified the significant objective of automobiles in long-term development. The electric automobiles will take place of the traditional fuel vehicles in next 15 years as well as step forward transportation electrification. However, the construction of charging infrastructure at present is still difficult in supporting large-scale automobiles to put on the market [2]. This is also the main cause to restraint users from turning to electric automobiles.

The obstacle to charge electric automobiles aggravated users' "anxiety on mileage". In addition, the uncertainty on the remainder of mileage, the energy consumption and else information plunged the automobile owners into travel anxiety frequently. It involved multiple factors to appraise whether the vehicle can reach destination in a certain travel. The aging of battery [3] and system failure [4] played a significant role in affecting the energy consumption. Based on the Internet of vehicles platform, Xiao W [5] portrayed the state of health (SOH for short) for large-scale automobiles, which indicated a giant difference in SOH among different areas and automobile models. Even if the same vehicle model with different operation years vary greatly in battery aging. This has great impact on available energy. In terms of energy consumption forecast, Yan J [6] divided the operating power consumption into speed energy consumption and accessories energy consumption, which were identified as per historical working conditions. Nevertheless, above algorithms require large amount of basic parameters from battery system or automobiles to build model and identify parameters. Thereupon they are not suitable for multiple vehicle models and various environmental conditions.

Depending upon the multi-network integration technology, this paper used the data driving method to analyse the dominant factors which affect the energy consumption and acquire the increased coefficient of power consumption in accordance with the historical operation data. Moreover, it invoked the path planning results of the map API to achieve energy consumption forecast and built the mapping relation of SOC and SOE. On the basis of above, it further calculated the potential demand for battery charging in this journey, thus realizing the charging demand alert.

\section{Energy consumption prediction and charging demand alert}

\subsection{Calculation on the increased energy consumption coefficient based upon the clustering and control variable methods under operation condition}

In the midst of actual driving on the road, the practical mileage will be reduced due to various external factors such as the road condition, driving speed, temperature and weather. In that the electric automobile won't consume energy while at idle, so when it brakes, the braking energy [7] can also recover at relatively greater rate. Therefore,

a Corresponding author: jiajun@ $@$ tsinghua-eiri.org 
traffic congestion has little impact on the increase of energy consumption for electric automobiles. On the other hand, when the automobile speeds up, it has to overcome more wind drag, thus consuming excess of energy. Furthermore, at relatively lower or higher temperature, the car owners have to switch on the air-conditioner, which apparently produces additional energy consumption. Consequently, the driving mileage reduces sharply. Again under extreme weathers, extra drag force occurs when the car body endures slaps of snow and rain. The resistance of roads grows as a result of rain and snow accumulation as well. However, such circumstances are not commonly seen. Thereupon, this paper chose driving speed and environmental temperature as the influential factors.

At first, the historical operation data of multiple vehicles with the same model within the same region were pre-treated. Then this paper extracted the long-term stable driving segment that possessed continual driving hours of no less than $T_{\min }$ and velocity series variance of no more than $\operatorname{Var}_{\max }$. Because the current was relatively stable under such working conditions, it was favorable to calculate the energy consumption. Here select $T_{\min }$ as 5 min and $V a r_{\max }$ as eight. Then obtain the sampling sequence of voltage, current and duration to calculate the mean value of voltage $\bar{U}$ within each sampling interval dt as well as the mean value $\bar{I}$ of current data. Finally obtain the energy consumption at operating section through the integral in Formula 1.

$$
W=\int_{0}^{T} \bar{U} \cdot \bar{I} \cdot d t
$$

The calculated mean speed and mean environmental temperature at each operating part was regarded as the feature description in this fragment. Among the acquired operating mode points, relatively more points were near to $50 \mathrm{~km} / \mathrm{h}, 60 \mathrm{~km} / \mathrm{h}, 70 \mathrm{~km} / \mathrm{h}$ and $80 \mathrm{~km} / \mathrm{h}$. This indicated that the automobiles run stably on most speed-limiting roads in the suburbs. Because the electric automobiles are not mainly used for trans-city or trans-provincial travel. Thereupon it's not so common to see high-speed operating conditions at more than $100 \mathrm{~km} / \mathrm{h}$ speed. The sample data of acquired automobiles mainly stemmed from SichuanChongqing region; The samples at below $0^{\circ} \mathrm{C}$ and above $42^{\circ} \mathrm{C}$ were comparatively fewer.

And then, regarding the mean velocity and average environmental temperature as the input parameters, the Mean-shift clustering algorithm was used to cluster the calculation fragment. The Mean-shift clustering algorithm [8] is a type of hill-climbing algorithm on the basis of nuclear density evaluation. It doesn't need to define the number of clusters artificially. Among the acquired clustering results, any operational fragments which possess adjacent speed and temperature are considered as the same type of operation condition. And the center point of each type is its characteristics of the operation condition. As shown in Figure 1, a number of characteristic conditions were acquired after clustering the working condition in this scope.

Calculate the average energy consumption of each operation segment in the same sub-category. Normally every mean energy consumption value was different from one another. Nevertheless, all of them follow the Gaussian distribution law. Calculate the normal distribution mean value $\mu$ as the mean energy consumption under this operating condition. If any particular abnormal point exists, remove it then continue calculation. From the calculated results, the divergence of average energy consumption of this car model was little at $40 \mathrm{~km} / \mathrm{h}$ speed within $[18,28]$ comfortable temperature. Furthermore, the value was closed to the energy consumption proposed by the Ministry of Industry and Information Technology. Thereupon, it could be used as the basic energy consumption $\mathrm{e}_{0}$.

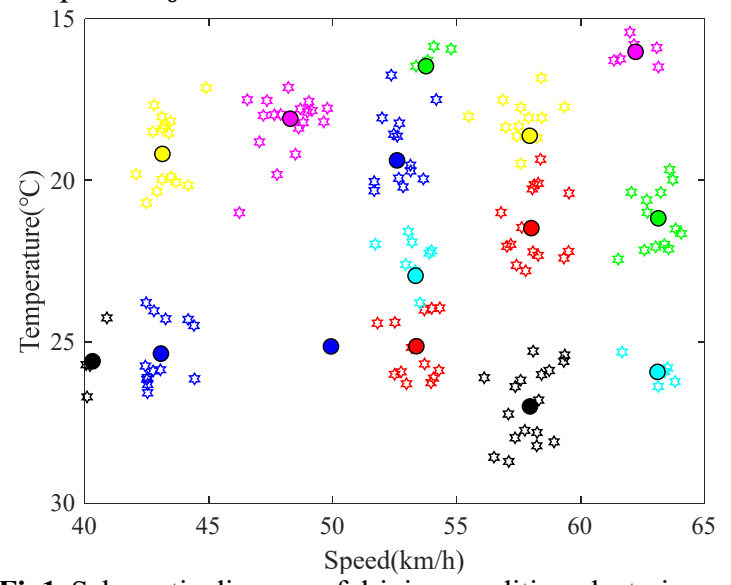

Fig1. Schematic diagram of driving condition clustering.

Ultimately, the method of variable control was used to calculate the impact of single factor on energy consumption increase. As the preferential temperature of human body is relatively broader, it exists a variety of actual operation modes. Control the temperature scope within $[18,28]$ first to get the growth coefficient $\left(\xi_{S}\right)$ of energy consumption at different speeds. Since the sample set was incomplete, part of the speed points cannot be covered. Accordingly, the Savitzky-Golay filter [9] was used to do interpolation on the discrete points. By sliding in the fixed window, this algorithm uses multinomial to realize the minimal double fitting inside the sliding window. It was shown in Figure 2.

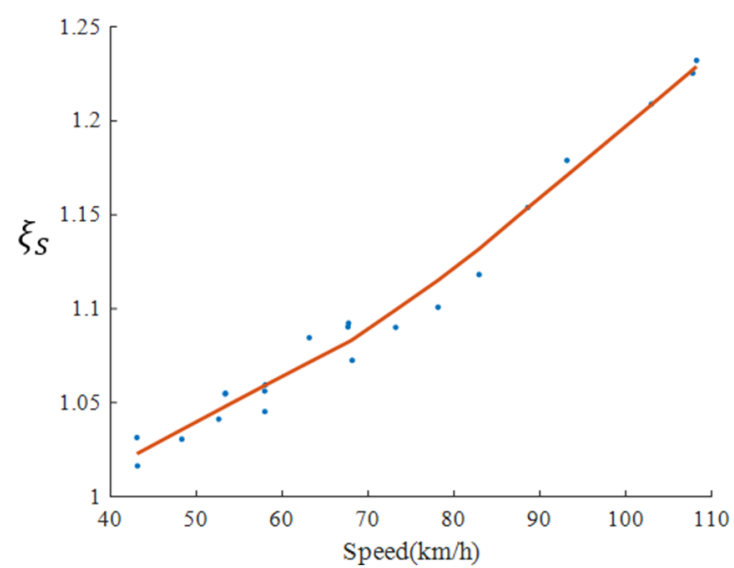

Fig2. $\xi_{s}$ Vs speed.

Set the speed as control variable at $5 \mathrm{~km} / \mathrm{h}$ scanning intervals. According to Figure 2, the power consumption at various speeds was converted into the energy consumption value at standard speed. It aimed to make the temperature as the only dependent variable of energy consumption changes. The growth coefficient $\left(\xi_{T}\right)$ of 
energy consumption at different temperatures was obtained. Its result was shown in Figure 3. In that the electric automobile doesn't have waste heat when it heats up, so it needs to rely on the heating of PCT component. Therefore, the gradient of energy consumption curve at low temperatures tends to be higher.

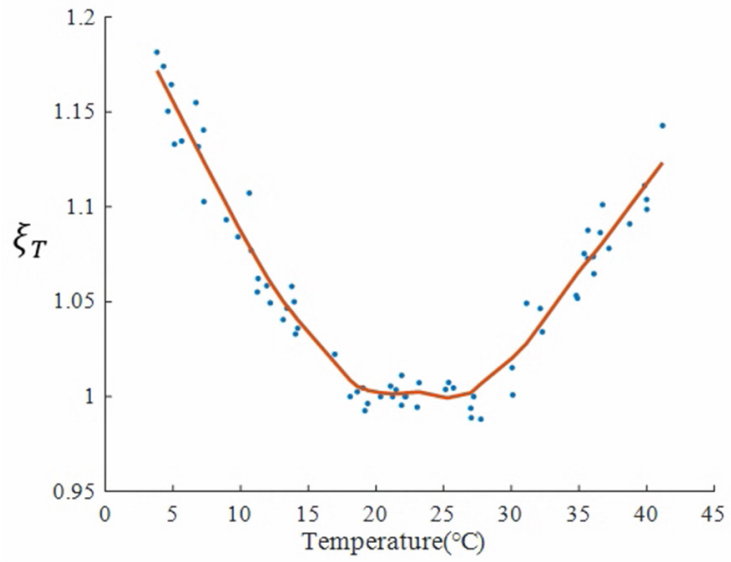

Fig3. $\xi_{T}$ Vs temperature.

Due to the limitation of objective conditions, this approach is only usable to control the scope of variables. In consideration of the error from data acquisition and data processing along with the extra consumption from car light, audio stereo and other accessories, the results consist of outlier value. So when fitting the curves, these factors should be eliminated.

\subsection{Power consumption prediction based upon the AMAP API}

The navigation path from the current spot to the target spot was obtained by map API. It consisted of the driving distances, congestion status, predicted driving duration and other information at every section. The prediction speed was then calculated to get corresponding growth coefficient of speed energy consumption. Through the weather interface, it could get the temperature information on every road section, consequently obtaining the corresponding growth coefficient of temperature and energy consumption. The corresponding distance at each section was: $\left[D_{1}, D_{2}, \ldots, D_{i}\right]$, so the prediction value of energy consumption on this road was:

$$
\begin{array}{r}
E n_{\text {pre }}=\left(\xi_{S, 1} * \xi_{T, 1} \cdot D_{1}+\xi_{S, 2} * \xi_{T, 2} \cdot D_{2}+\cdots+\xi_{S, i} *\right. \\
\left.\xi_{T, i} \cdot D_{i}\right) * \mathrm{e}_{0}
\end{array}
$$

\subsection{Evaluation on residual usable energy}

In the midst of automobile operation and charging, SOC is usually used to characterize the residual capacity of the battery. However, in the light of Formula 1, voltage is an indispensable part in calculating energy. Decided by the external characteristics of lithium battery, the voltage always changes in the process of charge and discharge. SOC is not linearly proportional with the residual energy. Therefore, in various voltage phases, the evenly spaced
SOC doesn't stand for the same energy output [10]. It was shown in Figure 4.

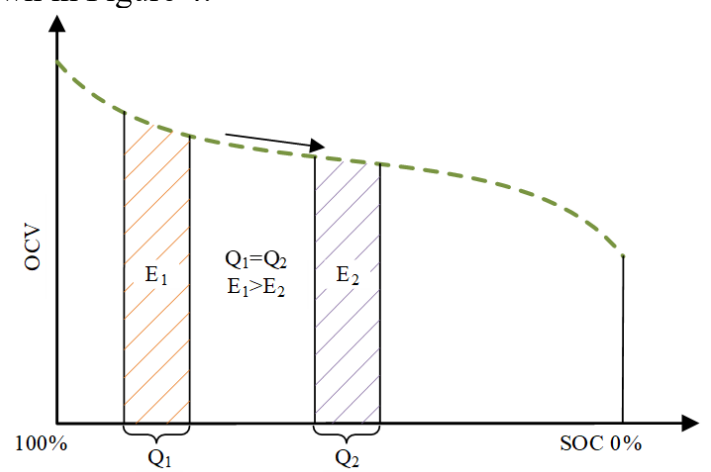

Fig4. The relationship between SOC-OCV curve and SOE

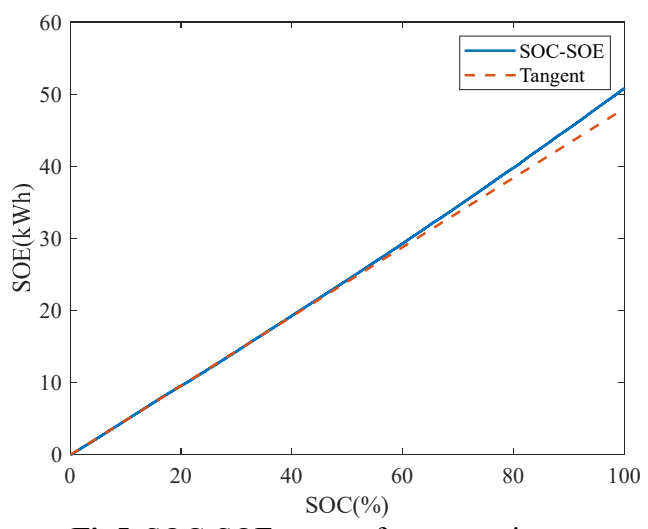

Fig5. SOC-SOE curve after correction

Additionally, as the voltage fluctuation of lithium battery is very susceptible to the charge-discharge rate, the battery voltage also changes suddenly at relatively higher rate. So, in actual operation environment, SOC cannot be simply used to calculate the coefficient of current residual energy. For this purpose, the multiple actual chargedischarge curves were extracted from the historical data by us to build the corresponding relation between SOC and SOE. The cases were as follows:

(1). Obtain the historical charging data fragment of target automobiles, then select the charging cycles which were comparatively late and complete.

The No. 3103 automobile was put into service on August 17, 2017. Its newest data were updated as of July 1,209 . There were 570 effective charging segments. From the analysis, in recent two years, this battery capacity of this automobile reduced $3.3 \%$. The recent energy output status was totally different from the circumstances of new cars. Therefore, when the calibration curves given by the manufacturer were used to calculate SOE, it didn't take into account the attenuation. Suppose the initial range of SOC was less than $20 \%$ and the due SOC was more than $80 \%$, then seven pieces of relatively intact charging fragments were searched out in the past month.

(2). Extract the significant data of voltage, current, SOC and else

(3). Calculate the sequence of charged energy in every cycle

(4). Make dynamic correction in accordance with a number of energy-charging curves to release the complete SOC-SOE curve. 
A variety of curves were used to complement the uncovered SOC scope so as to gain a complete SOC-SOE curve, which was shown in Figure 5. Given that the highvoltage section can release relatively more electricity, the gradient of curve slightly increased with the growth of SOC. After gaining the SOC-SOE curve, it only required to check corresponding SOE to obtain the remaining energy in accordance with current SOC. Although the variation of driving power might lead to unstable voltage in the process of real operation, constant-speed driving and parking usually took place on the way. Thereupon, under similar operation conditions, the SOC could be revised to further acquire accurate SOE after the voltage and current went stable.

\subsection{Judgement on charging demand}

Formula 3 defines the available energy:

$$
E n_{\text {available }}=S O E_{\text {current }}-S O E_{\text {destination }}
$$

In it, $S O E_{\text {destination }}$ represents the acquired $\mathrm{SOE}$ in accordance with SOC at the destination $\left(S O C_{\text {destination }}\right)$. $S O E_{\text {current }}$ represents the acquired SOE in accordance with current $\operatorname{SOC}\left(S O C_{\text {current }}\right)$. The available energy indicated the usable energy value after removing the user's expected energy at the destination. Formula 4 was used to calculate the needed charging energy:

$$
E n_{\text {charge }}=E n_{\text {pre }}-E n_{\text {available }}
$$

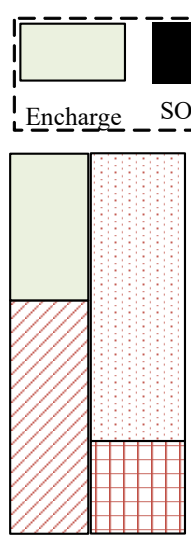

(a)
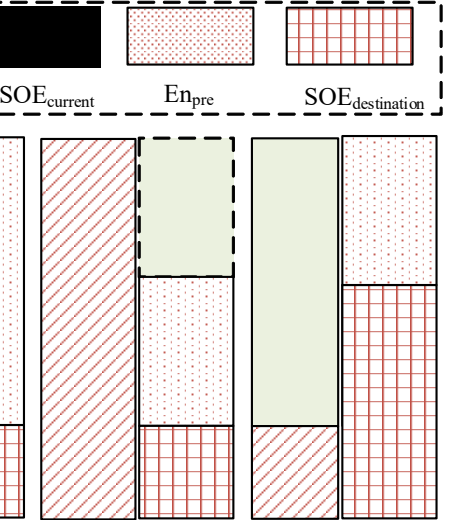

(b)

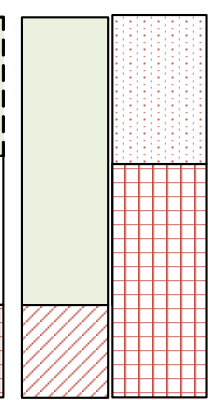

(c)
Fig6. Three situations of En charge.

Figure 6 corresponded three common scenarios: (a) indicated the circumstances when $S O E_{\text {current }}$ was larger than the $S O E_{\text {destination. Although the available energy }}$ was positive, it was insufficient to support the energy consumption; (b) indicated the circumstances when $S O E_{\text {current }}$ was sufficient to support the energy consumption and completely meet the $S O E_{\text {destination; }}$ (c) represented the circumstances when $S O E_{\text {current }}$ couldn't even meet the expected SOE for reaching the destination. Shown from the analysis, (a) and (c) circumstances required charging and energy to be charged was positive value. Ever since the user started a journey and every certain duration afterwards ( 5 minutes, for instance), the data platform would renew real-time data once. It calculated $E n_{\text {charge }}$. Once the requested charging energy was above zero, it would issue the charging demand alert.

\section{Case analysis}

A case analysis was presented as follows. For convenience in validation, set the expected $S O C_{\text {destination }}$ as $20 \%$. The automobile parameters and user demands were shown in Table 1:

Table 1. Vehicle parameters and user requirements

\begin{tabular}{|l|l|}
\hline Battery capacity & $50.83 \mathrm{kWh}$ \\
\hline $\begin{array}{l}\text { Starting } \\
\text { point }\end{array}$ & Chengdu Science City \\
\hline Destination & $\begin{array}{l}\text { Sandaoyan Ancient Town, } \\
\text { Pixian County }\end{array}$ \\
\hline \multicolumn{1}{|c|}{$\boldsymbol{S O C}_{\text {current }}$} & $45 \%$ \\
\hline Expected $\boldsymbol{S O C}$ destination & $20 \%$ \\
\hline
\end{tabular}

Three available paths were obtained by means of the AMAP API (The invoking time was 17:25 Oct. 28, 2020, the non-working day). After that, invoke the API of static map and drew Figure 7:

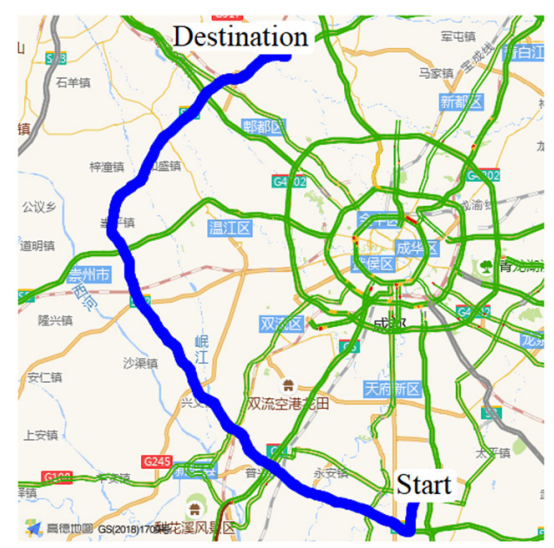

(a) path 1 ;

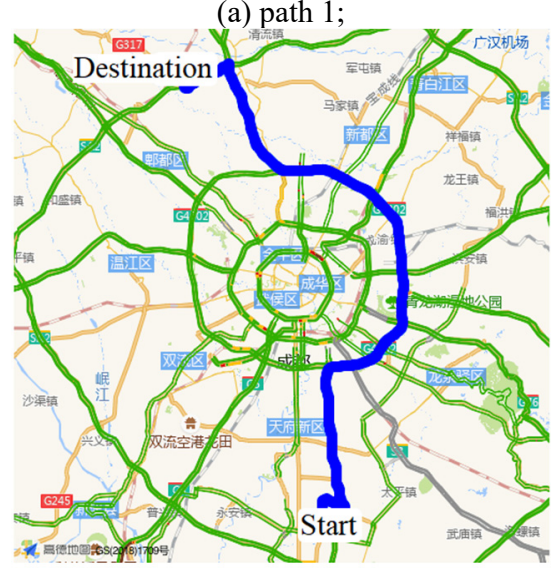

(b) path 2; 


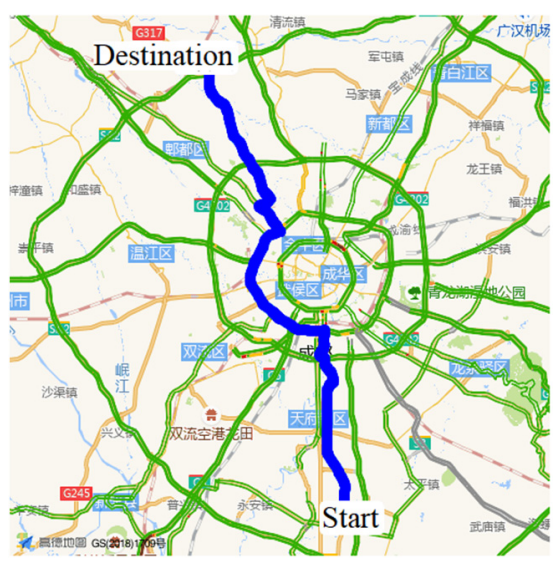

(c) path 3

Fig7. Three recommended paths obtained by map API

The comparative information of three paths was shown in Table 2:

Table 2. Comparison of three path information

\begin{tabular}{|l|l|l|l|}
\hline Path & $\mathbf{1}$ & $\mathbf{2}$ & $\mathbf{3}$ \\
\hline Distance (m) & 100368 & 91218 & 80210 \\
\hline Duration (s) & 4097 & 5673 & 6581 \\
\hline Number of traffic lights & 5 & 12 & 20 \\
\hline Congestion Index & 1 & 1.014 & 1.034 \\
\hline $\begin{array}{l}\text { Average energy consumptio } \\
\text { n forecast (kWh/100km) }\end{array}$ & 16.41 & 15.91 & 15.60 \\
\hline En pre (kWh) & 16.47 & 14.51 & 12.51 \\
\hline En $n_{\text {charge }}(\mathrm{kWh)}$ & 3.85 & 1.89 & -0.103 \\
\hline Charging demand & Yes & Yes & No \\
\hline $\begin{array}{l}\text { Predicted SOC destination } \\
(\%)\end{array}$ & 12.0 & 15.90 & 20.1 \\
\hline Actual SOC destination (\%) & 12.53 & 14.60 & 20.51 \\
\hline
\end{tabular}

The first path was nearly whole course of highway. Therefore; it had few traffic lights and it's the most timesaving. Nevertheless, now that the driving speed was too fast and the wind drag was relatively larger, the occurred mean power consumption was relatively greater. What's more, the overall mileage of the first path is comparatively longer; Although the third path had the shortest distance, it owned excessive traffic lights and congestion sections on the road. It took too much time. But it is worth noting that, the needed charging capacity of this path was less than zero. That was, it doesn't need charge. To validate the analysis, the same automobile drove for test on three paths under similar weather at rush hours. The $S O C_{\text {destination }}$ of three paths was shown in Table 2 . The error was respectively $4.23 \%, 8.90 \%$ and $2.0 \%$. The mean historical power consumption per hundred kilometers for the car was $15.72 \mathrm{kWh}$. If the mean energy consumption forecast method was used in prediction, the SOC $C_{\text {destination }}$ was $13.40 \%, 16.20 \%$ and $19.9 \%$; and the error was $6.94 \%, 10.96 \%$ and $2.97 \%$ respectively. The third path even mis-issued the charging demand alert. It could be seen that this approach can effectively increase the accuracy in comparison to traditional mean energy consumption forecast method and further provide reliable charging demand alert..

\section{Conclusion}

Supported by the massive data from multiple sources including automobiles, charging piles, map and transportation on the monitoring platform of new-energy automobiles, the clustering and control variable methods were used to realize energy consumption forecast and charging demand alert. It effectively relieved the user's travel anxiety accordingly. Moreover, with the accumulation of operation data for the same car models on the platform of the Internet of vehicles, the energy consumption forecast will be more and more accurate.

In consideration that the weather data wasn't included in the historical data pool, in the future of further study, the adverse weather and wind speed will be taken into account as impacting factors on the energy consumption. Meanwhile, the influence of rapid speed-up and abrupt deceleration as well as other driving habits will also be the focus of research.

\section{Acknowledgments}

The work is supported by the China Southern Power Grid Technology Project (0319002020030103JH00066).

\section{References}

1. Official website of the Ministry of Industry and Information Technology. The Ministry of Industry and Information Technology held a kick-off meeting for the preparation of the "New Energy Automobile Industry Development Plan (2021-2035)" [J]. People's Public Transport, (2): 27-27 (2019)

2. National Energy Administration. Annual Report of China Electric Vehicle Charging Infrastructure Promotion Alliance [EB/OL]. http://www.nea.gov.cn/2017-6/19/c_136376732.html

3. Xiao W, Xu H., Jia J*, Feng F, \& Wang W. State of Health Estimation Framework of Li-on Battery Based on Improved Gaussian Process Regression for Real Car Data. IOP Conference Series: Materials Science and Engineering, 793, 012063(2020).

4. Jia J, Deng Z, Hu X, et al. Data-driven comprehensive scoring of lithium-ion battery health status and abnormal battery screening $[\mathrm{J}]$. Chinese Journal of Mechanical Engineering, (to be published).

5. Xiao W, Zhong W, Shu X, et al. Estimation and application of battery state of health $(\mathrm{SoH})$ based on big data[J]. Journal of Automotive Safety and Energy, 10(01):105-109(2019)

6. Yan J, Xiao W, Jia J, et al. Remaining driving range data-driven algorithm based on energy consumption parameter identification and road condition prediction [J/OL]. Journal of Chongqing University of Technology (Natural Science): 1-13(2020)

7. Ma Z, Sun D . Energy Recovery Strategy Based on Ideal Braking Force Distribution for Regenerative Braking System of a Four-Wheel Drive Electric Vehicle[J]. IEEE Access, PP(99):1-1(2020) 
8. Chen H, Yang S, Pei J, et al. Collaborative filtering recommendation algorithm based on matrix factorization and Meanshift clustering $[\mathrm{J}]$. Computer Science and Applications, 010(004):P.649-658(2020).

9. Cai T, Tang H. Summary of the least square fitting principle of Savitzky-Golay smoothing filter[J].Digital Communications, 38(01):63-68(2011)

10. Hu X, Feng F, Liu K . State estimation for advanced battery management: Key challenges and future trends[J]. Renewable \& Sustainable Energy Reviews, 114(OCT.):109334.1-109334.13(2019) 